ARTICULOS ORIGINALES Rev Chil Salud Pública 2013 Vol 17 (1): 28-34

\title{
Efectos del megasismo del 27 de febrero de 2010 en Hospital Regional de Talca. Análisis bioético enfocado en la prevención
}

\section{RESUMEN}

Se realiza un análisis desde el punto de vista bioético de los efectos negativos causados en la atención sanitaria de la población usuaria del Hospital Regional de Talca, producto de los serios y extensos daños que este establecimiento sufrió luego del megasismo del 27 de febrero de 2010. Se utiliza un método de tipo deliberativo: análisis de los hechos (vulnerabilidad previa al sismo, daños ocurridos, estándares técnicos de sismo, resistencia de los establecimientos de salud), análisis de los valores involucrados y análisis de deberes, enfatizando la responsabilidad de prevención de daños de los establecimientos hospitalarios producto de terremotos, para asi facilitar la condición de posibilidad que permita llevar a cabo la respuesta sanitaria de urgencia y posteriormente las prestaciones habituales posterremoto.

Se concluye que debido a la alta sismicidad de nuestro territorio y a la posibilidad técnica de prevenir en gran medida los daños estructurales de los establecimientos hospitalarios, resulta de vital importancia que el Estado y la Sociedad chilena, incorporen como paradigma una Ética de la Responsabilidad, la que teniendo como fin la preocupación por la población vulnerable, aúne esfuerzos para que en lo posible siempre se hagan efectivas las medidas de normalización o de reconstrucción hospitalaria, a priori de los eventos telúricos

\section{Effects of the 'Mega' earthquake on February 27, 2010, on the Talca Regional Hospital. BIOETHICS ANALYSIS FOCUSED ON PREVENTION}

\section{ABSTRACT}

DAvid SChNettler

Cirujano Pediátrico. Profesor Instructor Facultad de Medicina, Universidad Católica del Maule. Estudiante de Magíster Interuniversitario de Bioética,

Universidad del Desarrollo 2011-2012.

Hospital Regional de Talca

"Dr. César Caravagno Burotto"

y Universidad Católica del Maule.

Dirección: 40 oriente 1548, Talca. 93285436. dcshnettlerrod@yahoo.com
A bioethics analysis was used to study the negative effects on health care among the population served by the Talca Regional Hospital, due to the serious, extensive damaged suffered by this hospital after the mega earthquake of February 27, 2010. A deliberative method is used: hard data was analyzed (preearthquake vulnerability, damage suffered, health center earthquake resistance standards), and analysis of values and responsibilities, emphasizing health center damage prevention responsibilities. The objective was to facilitate conditions that permit better implementation of emergency health care responses, and later, the restoration of routine health care, following an earthquake. It was concluded that due to the frequency of earthquakes in Chile, and technological advances that prevent of structural damages in health centers, it is of vital importance that the government and Chilean society incorporate an ethics model in order to protect vulnerable populations and make normalization and reconstruction efforts more effective in post-earthquake scenarios.

Key words: Disasters, earthquakes, bioethics, prevention, hospitals, responsibility. 


\section{INTRODUCCIÓN}

Los eventos de origen telúrico constituyen una realidad frecuente y recurrente en Chile. Las consecuencias de estos eventos para la población consisten en producción de lesiones o mortalidad, repercusiones psicológicas, pérdidas de tipo material y alteración de los servicios en general. En la atención de salud, suelen ocurrir desde deficiencias transitorias en la oferta sanitaria, hasta grave déficit de atención ocasionado por pérdidas estructurales, no estructurales y de alteraciones organizacionales.

El objetivo de este trabajo, es analizar desde el punto de vista bioético, las consecuencias para los habitantes de la Región del Maule ocasionadas por los severos daños que sufrió el Hospital de Talca debido al megasismo del 27 de febrero de 2010 (grado 8,8 en la escala de Richter, epicentro en la costa norte de la región del Bío Bío) ${ }^{1}$. Este artículo se centra en identificar la vulnerabilidad estructural previa de este recinto y determinar si existe la tecnología sismo resistente que nos permita evitar el daño estructural. A partir de estas premisas, se analizan los conceptos de prevención y responsabilidad, como estrategias que promuevan que el Estado y la Sociedad en general, se adelanten al daño de manera efectiva.

\section{MÉTODO DE ANÁLISIS}

Se detallan los efectos producidos en la población usuaria del Hospital Regional de Talca producto del megasismo del 27 de febrero de 2010, obtenidos de fuentes formales y de la propia vivencia del autor en su calidad de médico funcionario de este hospital. Se identifican documentos que hayan evaluado la vulnerabilidad sísmica del Hospital Regional de Talca, previamente al 27 de febrero de 2010, y los planes de renovación de la infraestructura de este hospital, por parte del Estado de Chile. Estos datos, se contrastan con recomendaciones internacionales sobre prevención de daño en instituciones de salud, ocasionadas por eventos sísmicos (OPS/OMS).

Para el análisis bioético, se utiliza una metodología deliberativa ${ }^{2}$, la que a partir de los hechos descritos, procede a analizar los valores y principios involucrados en esta catástrofe, para así discutir sobre los deberes que se generan a partir de ellos.

\section{DESCRIPCIÓN DE LOS HECHOS}

Efectos del megasismo del 27 de febrero de 2010 en el Hospital Regional de Talca y su población usuaria:

Este sismo dejó severamente dañados seis hospitales de la Región del Maule, estimándose que la mayoría de los hospitales de esta región quedaron funcionando a un $20 \%$ de su capacidad ${ }^{3,4}$. En el caso del Hospital Regional de Talca, inmediatamente después de este terremoto se implementaron hospitales de campaña gracias a aportes de instituciones públicas y privadas, nacionales $\mathrm{e}$ internacionales, y luego se planificó la construcción de hospitales modulares, los que comenzaron a funcionar en diferentes fechas posterremoto. A pesar que las autoridades nacionales y regionales han avanzado mucho en rehabilitar la infraestructura perdida, se mantendrá una alteración en la atención que se recuperará completamente al menos cuatro años después de la ocurrencia del terremoto, con el funcionamiento del nuevo hospital, actualmente en etapa de construcción.

Respecto a las medidas de reorganización, durante los primeras horas a semanas posterremoto, sólo se resolvió lo que fue posible, enfocándose principalmente en las situaciones de mayor urgencia vital, mientras la red hospitalaria pública y privada regional y nacional, absorbió la atención del resto de los pacientes graves. Las demás patologías, quedaron postergadas en este período.

La infraestructura posterremoto ha sido claramente insuficiente en espacios. El personal de salud ha manifestado sensaciones de frustración y cansancio, por trabajar en situaciones estresantes, que se prolongarán a mediano plazo.

Para la población usuaria, al pasar los meses ya no está presente el terremoto como justificación absoluta, y las exigencias son similares al período previo al terremoto. La vivencia diaria, es de practicar una medicina de excepción, pero las demandas son las de una comunidad no siniestrada, con presiones administrativas, de relaciones personales (entre funcionarios y con la población usuaria) y médico legales, diariamente.

Es muy probable que tal repercusión para la población, afecte negativamente los indicadores de salud y de desarrollo humano de la Región del Maule, en el mediano y largo plazo5. 
Estudios de vulnerabilidad sísmica del Hospital Regional de Talca previamente al 27 de febrero de 2010, y los planes de renovación de la infraestructura de este hospital por parte del Estado de Chile.

En la Región del Maule, varios hospitales de la región, incluido el Hospital Regional de Talca, presentaban conocidas vulnerabilidades frente a sismos de mayor magnitud, que hacían previsible un posible daño estructural mayor simultáneo, como ocurrió el 27 de febrero de $2010^{6-8}$.

El Hospital Regional de Talca "Dr. César Caravagno Burotto", fue construido por última vez el año 1937, luego de que fuera destruido sucesivamente por los terremotos de 1835 y 1928. Las estructuras más modernas de este hospital, datan del año 2005 -Clínica Pensionado- y 2007 Centro de Diagnóstico Terapéutico- (CDT) ${ }^{9,10}$.

El estudio de normalización del Hospital Regional de Talca (año 2000) ${ }^{10}$, reconoció como un aspecto crítico la vulnerabilidad de la estructura hospitalaria, que ya había sido dañada por el terremoto de 1985, y propuso su renovación.

El documento "Modelo De Gestión Para La Normalización. Hospital Regional de Talca", año 2007, reconoció "deficientes condiciones de trabajo, para desarrollar las acciones sanitarias a los usuarios, esto es, planta física en regular y mal estado, con baja funcionalidad entre servicios y unidades; equipamiento médico e industrial con obsolescencia tecnológica y vida útil agotada".

Posterior a estos estudios, el plan de renovación del Hospital Regional de Talca avanzó a la etapa de aprobación presupuestaria por parte del Estado, con el fin de iniciar la licitación pública para su construcción. Esta aprobación, sin embargo, sufrió varios retrasos y sólo fue aceptada luego de varias movilizaciones de los gremios de la salud locales, durante el año 2009, lideradas por el Colegio Médico Regional del Maule A.G. ${ }^{11}$. Sin embargo, esta renovación no alcanzó a materializarse antes del 27 de febrero de 2010, por lo que este terremoto dejó inutilizado prácticamente todo el edificio de 1937. La Clínica Pensionado del año 2005 también sufrió daños estructurales y debió ser evacuada. El CDT del año 2007, sufrió varios daños de tipo no estructural, siendo recuperada su función de forma progresiva en pocos días, constituyéndose en la primera construcción sólida en funcionar como recinto hospitalario.
Análisis de estándares y recomendaciones OPS/OMS sobre prevención de daño en instituciones de salud, ocasionadas por eventos sísmicos.

El documento de la Organización Panamericana de la Salud (OPS) "Fundamentos Para La Mitigación De Desastres En Establecimientos De Salud", (2004) $)^{12}$ reporta que los eventos naturales, en especial los de origen telúrico, constituyen una importante amenaza para la población de Latinoamérica. La vulnerabilidad está dada por elementos poblacionales, organizativos, y de las edificaciones hospitalarias.

La OPS, establece que la seguridad sísmica de los establecimientos de salud, debe mantener estándares que dependen del nivel de sismicidad de la región donde funcione el establecimiento. En el caso de Chile, donde el riesgo de sismicidad es $>50 \%$ para períodos de 30 años, se determina que posterior a un sismo, el establecimiento debe quedar inmediatamente operativo y sólo son aceptables fallas en áreas básicas como edificios de oficinas y residencias. Tanto las construcciones sismo-resistentes, como los planes de emergencia, deberían funcionar de manera óptima en estas situaciones. Esto es parte del concepto de hospital seguro $^{11}$. La recomendación, es que se planifique una vigilancia y planificación de mantención o renovación de elementos estructurales y no estructurales, en instituciones de salud vulnerables. El Estado Chileno adhiere a estas recomendaciones.

A modo de conclusión, el análisis de los hechos deja en evidencia que este establecimiento hospitalario presentaba conocidas vulnerabilidades estructurales, frente a las que existían planes concretos de renovación, pero que no se alcanzaron a materializar por atrasos presupuestarios. A raíz de la recomendación OPS/OMS, hay claridad de que a pesar de la gran magnitud del sismo ocurrido, sí existe la posibilidad técnica de construir establecimientos que resistan estos sismos y que permitan continuar con la atención de salud cuando más se necesita.

\section{DISCUSIÓN}

Análisis de los valores y principios involucrados:

Prosiguiendo con el método propuesto, el análisis valórico de la situación planteada nos permite reconocer valores que interesan a las 
instituciones de salud, al personal sanitario y a la población usuaria, como se detalla a continuación.

Desde el punto de vista de la atención pública de salud, los desastres naturales ponen en juego los principios de Justicia y No Maleficencia, entendiendo que el Estado debe facilitar la condición de posibilidad que permita una oportunidad de atención -acceso y calidad-con las adecuadas medidas de seguridad que estos procesos implican, incluyendo la seguridad de los establecimientos hospitalarios.

La visión de los profesionales y personal sanitario, además de la seguridad y del clima laboral, involucra que la instituciones les permitan ejercer una medicina de calidad, de acuerdo al estándar aceptado (Lex Artis).

La población usuaria, además del acceso y seguridad, valora recibir una medicina de calidad en todas sus dimensiones (prevención, promoción, diagnóstico, terapia, cuidados paliativos, etc.), en un contexto de adecuada relación clínica que favorezca la Beneficencia y Autonomía de las personas.

Ahora bien, producido el desastre, la jerarquía valórica sufre inmediatamente profundos cambios, ya que la integridad de la población viene a ser el primer valor a asegurar. Por este motivo, la prioridad en las primeras horas y días posterremoto, está en la optimización de recursos con el fin de atender a la mayor parte posible de la población siniestrada, muchas veces con criterios de triage ${ }^{13}$. En este nuevo escenario, la autonomía y el respeto a la confidencialidad, quedan supeditadas a las acciones beneficentes que los equipos de rescate e instituciones de salud puedan realizar.

El personal de salud queda situado tanto en el papel de víctima del desastre, como en el de auxiliador. Es por esto que el ejercicio de virtudes profesionales como la solidaridad, benevolencia, postergación de sí mismo, compasión, cuidado, imparcialidad y justicia, entre otras ${ }^{14,15}$, cobran extremada relevancia, ya que los escenarios de desgracia y caos, obligan a dar lo mejor de sí mismo para contribuir a la mayor beneficencia y justicia posible en la entrega de estas prestaciones.

Finalmente, si contrastamos la alta sismicidad de nuestro territorio con la gran cantidad de valores involucrados, cabe resaltar la actitud de previsión como valor fundamental para enfrentar la vulnerabilidad que nos caracteriza como país, frente a estos desastres.
Análisis de los deberes que se emanan de los hechos y valores involucrados: concepto de Responsabilidad.

¿Qué puede decir la Bioética respecto de la obligación de adelantarse a un evento como un terremoto, para así evitar consecuencias negativas en la atención de salud? Los hechos evidencian que el daño que actualmente se produce derivado de un terremoto, depende de la interacción de las características del sismo (factor no evitable) y de las medidas preventivas que podamos haber adoptado (construcciones, respuesta frente a desatres, etc.). Frente a la vulnerabilidad que presenta la población producto del posible daño en una institución hospitalaria, sobre todo existiendo la tecnología que nos permita mitigar estos daños, aparece como respuesta obvia la necesidad de una Ética de la Responsabilidad, que nos ayude a enfrentar estos desafíos. Este concepto no debería entenderse solamente como ligado a la culpa -no se trata sólo de responder a posteriori de los hechos ya ocurridos- sino más bien a una responsabilidad relacionada con la solidaridad, que nos permita como sociedad organizarnos de manera que todas las acciones tecnológicas tiendan a la beneficencia de la mayoría de las personas ${ }^{16}$.

¿De qué maneras se puede ejercer esta responsabilidad? La prevención -antiguo concepto derivado de la realización de la virtud aristotélica de la prudencia- en el escenario descrito aconsejaría tomar medidas que permitan adelantarse al daño cada vez que sea posible, cuando una persona o población está expuesta a peligros derivados de desastres naturales. La prevención se aplica ampliamente en ámbitos de seguridad laboral, sin embargo, en relación a desastres naturales y efectos negativos derivados de la acción humana, el desarrollo de este concepto como obligación imperativa, es menor.

La actitud de previsión no se restringe a temas biomédicos solamente, ya que implica consecuencias de la aplicación de tecnologías, tales como energía nuclear, contaminación ambiental, manipulación genética, etc. Para estas temáticas, en que existe incertidumbre de las consecuencias derivadas de la acción humana, se gestó el principio de precaución, que ha venido discutiéndose y analizándose desde hace varias décadas, planteándose como principio por primera vez en Alemania, el año 1974 (término Vorsorgeprinzip) $)^{17}$, siendo internacionalizado el año 1992, en la Conferencia 
de las Naciones Unidas, Río de Janeiro ${ }^{18}$. Este principio, se refiere a las medidas cautelares que pudieran aconsejar tomar precauciones u omitir una acción, frente a riesgos derivados principalmente de la acción humana, en un escenario de incertidumbre o de ignorancia de posibles efectos nocivos, a corto o largo plazo ${ }^{19}$.

El término prevención propiamente tal, es más aplicable a la situación analizada, ya que se refiere a las evaluaciones y acciones destinadas a prevenir o mitigar un daño, derivado de riesgos conocidos (sismos), e impactos esperables (daño estructural, vidas humanas, elementos organizacionales, etc. $)^{20-}$ ${ }^{22}$. La prevención obliga más que la precaución en vista del nivel de desarrollo tecnológico actual.

¿Quién es responsable de aplicar estas medidas? La prevención, es parte importante de la responsabilidad de protección de los Estados, según ha declarado las Naciones Unidas en su informe sobre Intervención y Soberanía Estatal (prevención, reacción y reconstrucción $)^{23}$. El Estado de Chile, debe regular y vigilar la entrega de prestaciones en salud, según consta en la actual Constitución Política de Chile, que se refiere al derecho a la protección de la salud (artículo 9) ${ }^{24}$. Sin embargo, por extensión todos los agentes sociales involucrados en los desastres -"stake holders"-, participan en alguna medida de esta responsabilidad.

Estas acciones se deberían enfocar en el desarrollo de estrategias de alerta temprana y respuesta a desastres $^{25-27}$, así como a asegurar la sismo-resistencia de la infraestructura hospitalaria. Los marcos referenciales para llevar a cabo una planificación preventiva y de respuesta frente a desastres, con especial interés en el impacto producido en la población afectada, se recoge en las directrices IASC que contrastan los desastres con la Declaración Universal de Derechos Humanos ${ }^{5}$ y el proyecto ESFERA $^{28}$, que incorpora nociones de calidad y de rendición de cuentas en las respuestas humanitarias.

\section{CONCLUSIONES}

Si bien es cierto un desastre natural no tiene valor moral en sí mismo, sí lo tienen las acciones humanas destinadas a preveer y prevenir en la medida de lo posible la vulnerabilidad frente a estos desastres. Pero las estrategias sociales para desarrollar acciones prudentes no son tan obvias y quizás en casos de desastres de gran magnitud, resulten imposibles de llevar a cabo de manera totalmente satisfactoria. No se puede concluir que en el caso analizado no existieron medidas precautorias. Por el contrario, la sociedad chilena ha debido enfrentar múltiples terremotos en su historia y es indiscutible su larga tradición de prevención, respuesta y recuperación frente a estos desastres naturales, con un número de víctimas fatales relativamente bajo comparado con otras zonas del planeta ${ }^{29}$.

Sin embargo, resulta llamativo que el Hospital Regional de Talca $-\mathrm{y}$ varios establecimientos públicos en la historia de nuestro país- sólo se terminen renovando cuando son destruidos por un terremoto, a pesar de las medidas de evaluación y planificación de reconstrucción por parte del Estado de Chile.

Existiendo múltiples factores que explican las causas de la dilación de estos procesos, entre ellos el alto costo de la reconstrucción de estos establecimientos y el hecho de competir presupuestariamente con decenas de otros proyectos de alto costo e impacto a nivel país, la propuesta bioética no apunta a las razones técnico-administrativas, ni legales, más bien se enfoca en reconocer que los valores involucrados en estos desastres, involucran a personas concretas y que la funcionalidad de un establecimiento de salud posterior a un desastre natural, constituye la condición de posibilidad para que se desempeñe la respuesta sanitaria de urgencia y posteriormente las prestaciones habituales.

El llamado es a destacar que el enfoque derivado de una ética de la responsabilidad, si bien difícil en su realización, significa un cambio de mirada que va más allá del deber, ya que busca responder a la vulnerabilidad de las personas en todos los ámbitos. Una ética de la responsabilidad podría tal vez permitir que éste y otros problemas similares, sean abordados por parte del Estado de Chile y la sociedad en general, de una manera que promueva que las acciones se lleven a cabo de manera oportuna.

\section{Agradecimientos}

A mis profesores del Programa de Magister Interuniversitario de Bioética Universidad del Desarrollo-Clínica Alemana, por alentarme a realizar este trabajo, en especial al Dr. Juan Pablo Beca Infante, jefe de programa, y a la Dra. María Inés Gómez Bradford, revisora de este proyecto de investigación. 


\section{REFERENCIAS}

1. US. Geological Survey [en línea]. U.S.: Department of the Interior, Geological Survey; [actualizado February 06, 2013; [consultado el $2 /$ 7/2012]. 2010 February 27 06:34:14 UTC. Disponible en: http://earthquake.usgs.gov/ earthquakes/eqinthenews/2010/us2010tfan/.

2. Gracia D. Teoría y práctica de la deliberación moral. En: Gracias D, Sánchez M. Bioética: el estado de la cuestión; Madrid: Triacastella; 2012. p. 101-154.

3. Allende S. Secuelas del terremoto en los hospitales. Rev Vida Médica [en línea]. 2010; (1): 12-18 [consultado el 11/3/2013]. Disponible en: http:/ /www.colegiomedico.cl/Portals/0/files/biblioteca/ publicaciones/revista_medica/vida_medica_2010_n1.pdf.

4. Sagredo M. Especial reconstrucción: radiografía hospitalaria a un año del terremoto. Rev Vida Médica [en línea]. 2010(1):6-12 [consultado el 11/3/2013. Disponible en: omedico.cl/Portals/0/ files/biblioteca/publicaciones/revista_medica/ vida_medica_2011_n1.pdf.

5. Instituto Nacional de Derechos Humanos (Chile). Estudio sobre la reconstrucción post terremoto desde una perspectiva de derechos humanos [en línea]. Santiago: INDH; 2012 [consultado en noviembre 2012]. Disponible en: «http:// www.indh.cl/wp-content/uploads/2012/07/ informe-reconstruccion-indh.pdf.

6. Boroschek R, Astroza M., Osorio C, Kausel E. Análisis de vulnerabilidad y preparativos para enfrentar desastres naturales en hospitales de Chile. Chile. Santiago, Universidad de Chile; Fundación para la Transferencia Tecnológica; 1996.

7. Boroschek R, Astroza M, Osorio C. Capacidad de respuesta de hospitales ante desastres sísmicos: aspectos no estructurales. Conferencia Internacional sobre Mitigación de Desastres en Instalaciones de Salud, OPS, México; 1996.

8. Chile. Ministerio de Salud. Guía de Vigilancia Epidemiológica de Emergencias y Desastres [en línea]. Santiago: OPS; MINSAL; 2010 [consultado el 2/7/2012]. Disponible en: 1/ALTA.pdf»http:// www.seremidesaludbiobio.cl/ALTA.pdf.

9. Servicio de Salud del Maule (Chile). Modelo de gestión para la normalización, Hospital Regional de Talca [en línea]. Talca: MINSAL; 2007 [consultado el 2/7/2012]. Disponible en: // www.hospitaldetalca.cl/2012/bd_documento/ autogestion_2012_03_08_32.pdf

10. Servicio de Salud del Maule (Chile). Estudio de Normalización Hospital Regional de Talca. Talca: MINSAL; 2000.

11. Diario El Mercurio [en línea]. 300 médicos paralizan por 48 horas por presupuesto de nuevo hospital de Talca [consultado el 2/7/2012]. http:/ /www.emol.com/noticias/nacional/2009/10/08/ 379273/300-medicos-paralizan-por-48-horaspor-presupuesto-de-nuevo-hospital-de-talca.html.

12. Organización Panamericana de la Salud. Fundamentos para la mitigación de desastres en establecimientos de salud [en línea]. Washington, D.C.: OPS; 2004 [consultado el 2/7/2012]. Disponible en: .paho.org/spanish/dd/ped/ Fundamentos-intro.pdf.

13. León FC. Después de un terremoto: bioética en situaciones de catástrofe. Rev Med Chile. 2012; 140: 108-112.

14. Beca JP. Problemas éticos en situaciones de catástrofes [en línea]. Santiago: Universidad del Desarrollo, Centro de Bioética; 2010 [consultado el 2/7/2012]. Disponible en: http:// medicina.udd.cl/centro-bioetica/files/2010/10/ parral.pdf.

15. Pellegrino E. Toward a virtue-based normative ethics for the health professions. Kennedy Inst Ethics J. 1995; 5(3): 253-77.

16. Feito L. La responsabilidad como clave de la ética actual. En: Beca JP, Astete C. Bioética clínica. Mediterráneo: Santiago de Chile; 2012. p. 50-61.

17. Comisión Mundial de Ética del Conocimiento Científico y la Tecnología (COMEST). Informe del Grupo de Expertos sobre el Principio Precautorio. Paris: Unesco; COMEST; 2005.

18. Organización de las Naciones Unidas. Declaración de Río sobre el medio ambiente y el desarrollo. Programa 21 [en línea]. Naciones Unidas [consultado el 2/7/2012] Disponible en: http:// www.un.org/spanish/esa/sustdev/agenda21/ riodeclaration.htm

19. Bonamigo EL. El principio de precaución: un nuevo principio bioético y biojurídico [tesis dcotoral en línea] [España]: Universidad Rey Juan Carlos. Dpto. de Ciencias de Educación, Cultura y Lenguaje; 2010. [consultado el 2/7/2012]. 
Disponible en: http://www.sbbioetica.org.br/ antigo/arquivos/Bonamigo\%20-\%20Tese.pdf.

20. Agencia Europea de Medio Ambiente. Lecciones tardías de alertas tempranas: el principio de cautela, 1896-2000: algunos puntos a modo de resumen. Dinamarca: AEMA; 2002.

21. Tickner J. A common sense framework for operationalizing the precautionary principle. Wingspread Conference on Strategies for Implementing the Precautionary Principle; 1998 23 al 25 de enero; Racine, Wisconsin.

22. Kottow M. Bioética en situaciones de catástrofe. Rev Chil Salud Pública. 2010; 14(1): 46-51.

23. International Commission on Intervention and State Sovereignty. The Responsibility to Protect [en línea]. Canadá: International Development Research Centre; 2011 [consultado el 2/7/2012]. Disponible en: http://responsibilitytoprotect.org/ ICISS\%20Report.pdf.

24. El derecho a la protección de la salud, Art. $19^{\circ}, \mathrm{N}^{\circ} 9$. Constitución Política de la República de Chile (1980).

25. White DB, Katz MH, Luce JM, Lo B. Who should receive life support during health emergency?: using ethical principles to improve allocation decisions. Ann Int Med. 2009; 15: 132-138.

26. Good L. Ethical decision making in disaster triage. J Emerg Nurs. 2008; 34(2): 112-5.

27. The Israeli Field Hospital in Haiti. Ethical dilemmas in early disaster response. NEJM 2010; 362: e 38.

28. El Proyecto Esfera: Carta humanitaria y normas mínimas para la respuesta humanitaria [en línea] [consultado en noviembre de 2012]. El Proyecto Esfera en breve. Disponible en: http:// www.sphereproject.org/sphere/es/acerca/.

29. US. Geological Survey [en línea]. US.: Department of the Interior, Geological Survey [actualizado febrero 06, 2013; consultado el 2/7/ 2012]. Earthquakes with 1,000 or More Deaths since 1900. Disponible en: http:// earthquake.usgs.gov/earthquakes/world/ world_deaths.php. 